Andreea Madalina Panciu ${ }^{1}$, Sandu Magdalena², Diana Epure ${ }^{2}$, Daniela Vasile ${ }^{2}$, Smaranda Nita $^{2}$, Roza Eugenia ${ }^{2}$, Raluca Teleanu$^{2}$,

${ }^{1}$ National Institute of Infectious Diseases "Prof. Dr. Matei Bals", Bucharest

'Clinical Children's Hospital "Prof. Dr. Victor Gomoiu"

Dr.Victor Gomoin
Rare patients infected with EBV Epstein-Barr virus) develop a lifethreatening condition termed Chronic active Epstein-Barr infection.

\section{Chronic active Epstein-Barr infection} $($ CAEBV $)=$

progressive lymphoproliferative disorder with a duration of $>6$ months, characterized by:

markedly elevated levels of antibody to EBV /or EBV DNA in the blood infiltration of tissues with lymphocytes

The absence of any other immunosuppressive condition elevated EBV-DNA ,RNA or protein in affected tissues

The only treatment reported successfu in literature is allogeneic HSCT hematopoietic stem transplantation)

\section{cell}

We present a rare case of a $\mathbf{1 2}$ year old. girl, with a history of:

Age month - Acute EBV temporal ( clinically - no new mononuceosis with hemofagocitic sign). lymphohistiocytosis and fulminant 11 years ( August) : right acute liver failure.

- 3 years - Abdominal CT show enlarged liver and spleen, no adenopathies ;

+ Liver biopsy $\Rightarrow$ Chronic viral hepatitis.

- 4 years - She starts presenting motor deficit cerebral CT- right and temporal myelination deficit cerebal MRI

disseminated infratentorial and supratentorial inflammatory lesions.

- 5 years - Spastic triparesis, strabismus bilateral VI nerve palsy, swallowing difficulties, gait disturbance, dysarthria

- treated with steroids; mild sequelae;

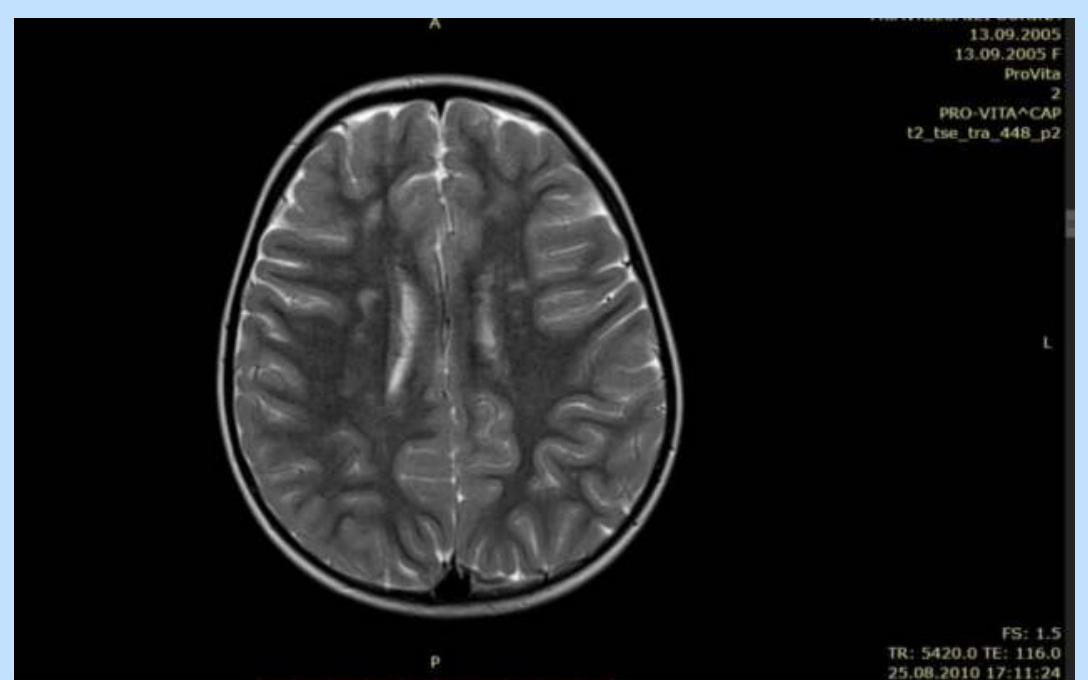

10 years - cerebral MRI for neurological monitoring ( 2015): sequelae of demyelination lesion; the largest one -left hemiparesis affecting mostly the right arm -head and cervical spinal cord MRI: new demyelinating lesions both encephalic and spinal cord favorable outcome with i.v steroid pulse therapy and then 9 months orally;

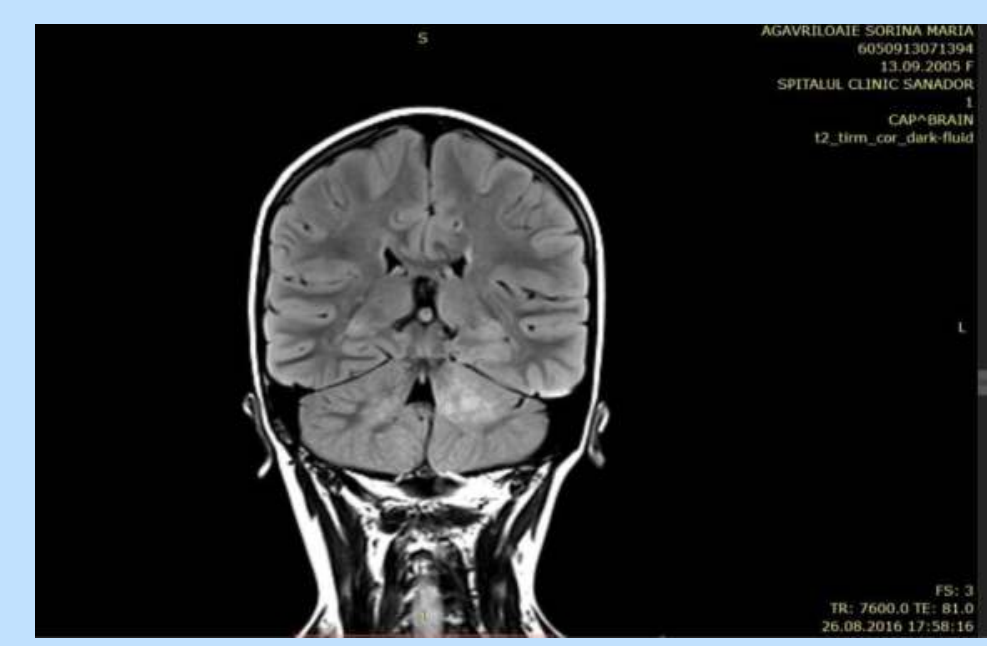

- 11 years (January) - cerebral MRI and cervical spinal cord reduced preexisting demyelinating lesions; new gadolinophilic and hemosiderin lesions in the cervical spinal cord

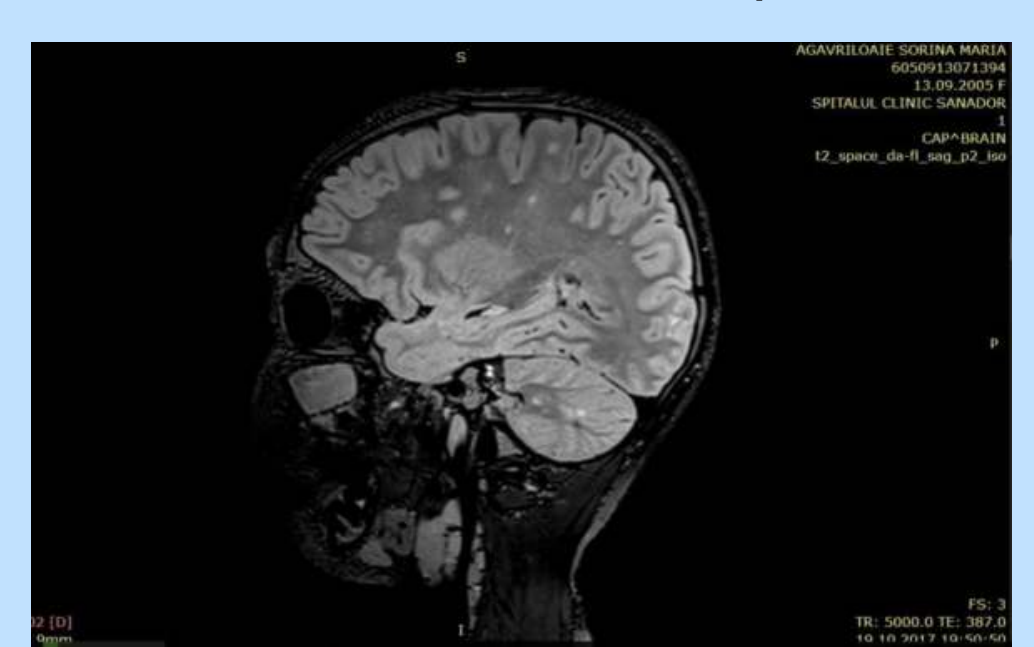

- 12 years- walking difficulties cerebral and spinal cord MRI important extension of the lesions

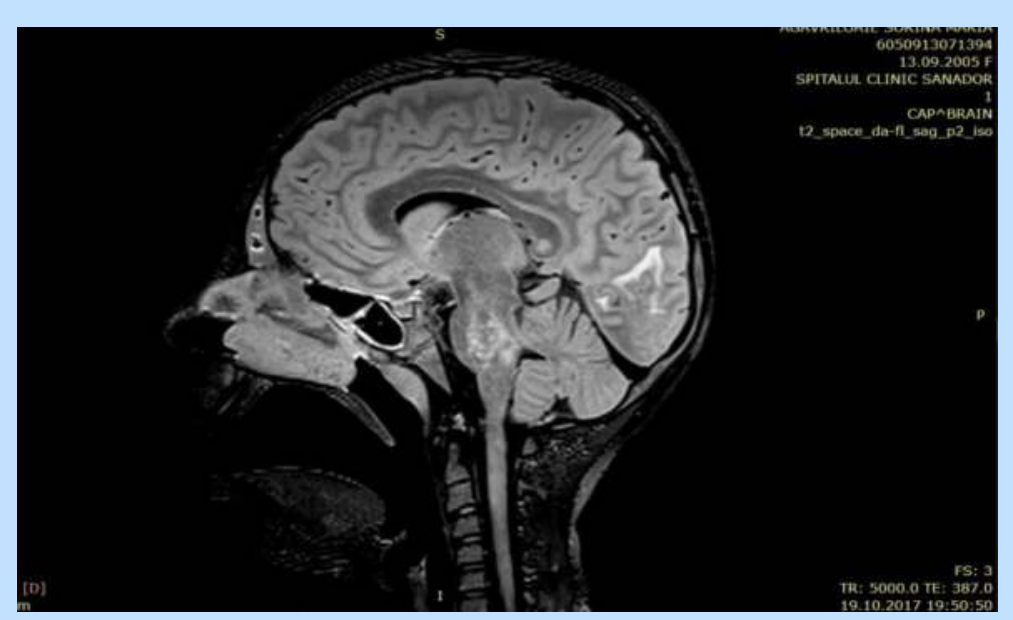

Clinical and

examination show:

paraclinical

Constant:

- Markedly elevated levels of antibodies to EBV in the blood

- Hepatosplenomegaly

- WBC around 3000/mm $\mathrm{m}^{3}$

- Low platelet number

- Mild anemia

- Moderate increase of liver enzymes

- Negative oligoclonal bands in CSF.

In conclusion, in the evolution of an infection with EBV, CAEBV may develop with the possibility of further neurological MS-like syndrome being associated. 